Original Article

\title{
Muscle activation of drivers with hemiplegia caused by stroke while driving using a steering wheel or knob
}

\author{
Nam-Hae Jung ${ }^{1)}$, Hwanhee Kim²), Moonyoung Chang ${ }^{3)^{*}}$ \\ 1) Department of Occupational Therapy, Baekseok University, Republic of Korea \\ 2) Department of Occupational Therapy, Semyung University, Republic of Korea \\ 3) Department of Occupational Therapy, College of Biomedical Science and Engineering, Inje \\ University: 197 Inje Street, Gimhae, Gyeongsangnam-do 621-749, Republic of Korea
}

\begin{abstract}
Purpose] The aim of this study was to investigate three muscle activities of drivers with post-stoke hemiplegia while they were driving using a steering wheel or a spinner knob, and to compare them with those of non-disabled drivers. [Subjects and Methods] The subjects were four non-disabled drivers and five drivers with left hemiplegia. The subjects drove forward in a straight line for $5 \mathrm{~m}$ and then turned right or left using the steering wheel or spinner knob with only their right hand. EMG electrodes were placed over the anterior deltoid, biceps and triceps brachii on the right-side. [Results] While differences in muscle activation between the spinner knob and the steering wheel in the control group were not significant, those of the experimental group were significant. Activation of the biceps brachii while the control group turned the vehicle to the right using the spinner knob was significantly lower than when using the steering wheel. Activation of the biceps brachii while the experimental group turned the vehicle to the right using the spinner knob was significantly lower than that of the control group. [Conclusion] The results of this study indicate that a spinner knob requires less activation of the main muscle than a steering wheel, especially in drivers who have had a stroke. The results could be used as basic data when driver rehabilitation specialists prescribe the spinner knob for patients.

Key words: Driving, Muscle activation, Spinner knob
\end{abstract}

(This article was submitted Oct. 2, 2014, and was accepted Nov. 28, 2014)

\section{INTRODUCTION}

Drivers spend a lot of time operating a steering wheel. Drivers operate a steering wheel using either both hands or one hand. Some drivers use a spinner knob instead of a steering wheel. Muscle activation varies according to the size and shape of the driver's hand grip ${ }^{1-3)}$ and it affects the amount of discomfort and fatigue they experience while they are driving.

People with post-stoke hemiplegia cannot use both hands while driving. Most of them use a spinner knob with their non-affected hand instead of a steering wheel. A spinner knob helps a driver control the steering wheel with only one hand. The number of drivers that have experienced a stroke is increasing ${ }^{4)}$. However, there is a lack of research about analyzing the driving performance of drivers who have had a stroke. Therefore, the purpose of this study was to investigate the muscle activates of drivers with post-stoke hemiplegia

*Corresponding author. Moonyoung Chang (E-mail: myot@ inje. ac.kr)

(C2015 The Society of Physical Therapy Science. Published by IPEC Inc. This is an open-access article distributed under the terms of the Creative Commons Attribution Non-Commercial No Derivatives (by-ncnd) License $<$ http://creativecommons.org/licenses/by-nc-nd/3.0/> . while driving using a steering wheel and a spinner knob, and compare them with those of non-disabled drivers.

\section{SUBJECTS AND METHODS}

While 10 participants were originally selected for this study, a total of nine drivers participated in this experiment: five drivers with post-stroke hemiplegia (experimental group) and four non-disabled drivers (control group). The stroke group did not have any neurological, musculoskeletal, cognitive, or perceptual problems except for the stroke. In terms of driving history, they had been driving for more than three years after they had a stroke. The control group consisted of four drivers of a similar age and gender as the experimental group. However, one driver in the control group elected to drop out of the study during the experimental process. This study was conducted with 3 males and 2 females in the experimental group and 3 males and 1 female in the control group. The average height of the experimental group was $163.5 \mathrm{~cm}$, and that of the control group was $162.75 \mathrm{~cm}$, and their average weights were 60.75 and $59.25 \mathrm{~kg}$, respectively. The average driving experience of the experimental and control groups was 15 years and 6 months, and 14 years and 5 months, respectively. The subjects participated in this study after receiving an explanation of purpose of this study and had signed a consent form. This research was approved 
by the Inje University's Institutional Review Board.

The Hyundai AVANTE was used as the experimental vehicle in this study. The driver's seat angle was $105^{\circ 5}$ and the height of the seat was $20.8 \mathrm{inch}^{6}$. A researcher adjusted the distance between the driver and the steering wheel to maintain a $113^{\circ}$ angle of the elbow when the participant held the steering wheel at a $30^{\circ}$ angle to the right from the midline $^{7)}$ (Fig. 1). A spinner knob was set at an angle $30^{\circ}$ clockwise from the center of the upper part of the steering wheel because the drivers in the experimental group had post stroke, left side hemiplegia. The speed limit was at least $10 \mathrm{~km} / \mathrm{h}$ but not more than $20 \mathrm{~km} / \mathrm{h}$. The experimental task consisted of driving forward in a straight line for $5 \mathrm{~m}$, turning right or left, and driving straight ahead for another $5 \mathrm{~m}$. The participants in the experimental group and the control group were instructed to only use either the steering wheel or the spinner knob with only their right hand.

Muscle activities were measured using surface EMG (TeleMyo $^{\text {TM }}$ 2400T G2, Noraxon USA, Inc., Scottsdale, AZ, USA). The collected surface EMG signals were converted into digital signals by a TeleMyo 2400T G2, and the converted signals were processed using MyoResearch Master XP 1.07 software. The EMG signal sampling rate was $1,000 \mathrm{~Hz}$. Electrodes were placed over the anterior deltoid, biceps brachii and triceps brachii on the right-side according to the method suggested by Mo et $\mathrm{al}^{8)}$. The EMG data were converted to root-mean-square values that provided values

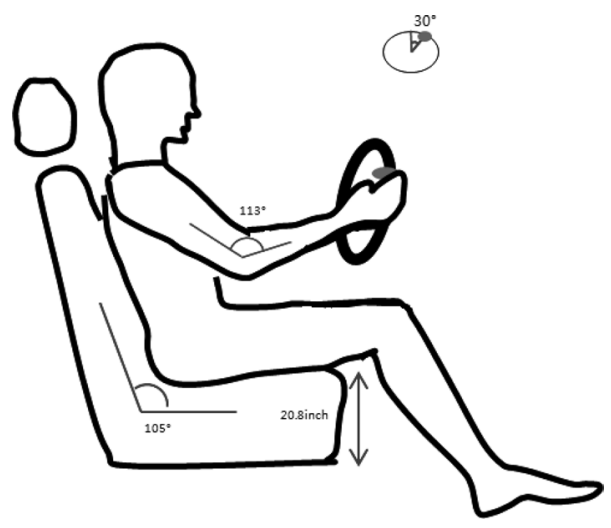

close to the actual output values of the EMG signals. For quantification, the EMG signal volumes as a percentage of the reference voluntary contraction ( $\% \mathrm{RVC})$ were measured. The reference voluntary contraction was measured in a sitting posture with the knees and hips flexed at $90^{\circ}$. Before beginning the experiment, EMG was measured when the participant flexed the elbow and then flexed the shoulder, extending the elbow with a 2-kg dumbbell in the hand. All the measurements were repeated three times and the average values were calculated.

Data were analyzed using SPSS 18.0. To test the homogeneity of age, gender, driving history, car model, and driver licenses classification between the experimental group and the control group, the $\chi^{2}$ test was performed. To compare activation of the three muscles between the two groups, the Mann-Whitney U test was performed. To compare the muscle activation between the spinner knob and the steering wheel by group, the Wilcoxon singed-rank test was conducted.

\section{RESULTS}

The results of the comparison of the activation of the three muscles between the steering wheel and the spinner knob while the drivers in the experimental group turned the vehicle to the left were as follows. There was no significant difference in the activation of the three muscles between the spinner knob and the steering wheel when drivers in the experimental group turned to the left. When the drivers in the experimental group turned to the right, a significant difference in the activation of the muscles between the spinner knob and the steering was found only in the biceps brachii.

The activities of the three muscles between the steering wheel and the spinner knob when the control group turned the vehicle to the left showed no significant differences. When the control group turned the vehicle to the right, there were no significant differences in activities of the three muscles between the spinner knob and the steering wheel. Activation of the biceps brachii while turning the vehicle to the right with the spinner knob was significantly lower in the experimental group than in the control group (Table 1).

Fig. 1. Driving posture

Table 1. Muscle activation during left and right turns made with a spinner knob and a steering wheel $(\mathrm{n}=10)$ (Unit: \% RMS)

\begin{tabular}{lccccc}
\hline & & \multicolumn{2}{c}{ Stroke } & \multicolumn{2}{c}{ Control } \\
\cline { 3 - 6 } & & Spinner knob & Steering wheel & Spinner knob & Steering wheel \\
\hline Turning to & Anterior deltoid & $27.1 \pm 9.1$ & $27.2 \pm 5.2$ & $35.0 \pm 11.7$ & $38.3 \pm 12.5$ \\
the Left & Biceps brachii & $17.8 \pm 1.7$ & $15.9 \pm 4.1$ & $22.9 \pm 10.3$ & $37.5 \pm 4.7$ \\
& Triceps brachii & $22.3 \pm 10.0$ & $20.3 \pm 9.1$ & $38.8 \pm 3.6$ & $36.6 \pm 6.7$ \\
Turning to & Anterior deltoid & $25.8 \pm 5.6$ & $26.1 \pm 3.9$ & $31.2 \pm 12.7$ & $32.9 \pm 16.7$ \\
the Right & Biceps brachii & $15.1 \pm 3.2^{*}$ a & $22.1 \pm 3.8^{\text {a }}$ & $38.7 \pm 9.9^{*}$ & $36.2 \pm 8.2$ \\
& Triceps brachii & $22.9 \pm 8.1$ & $26.9 \pm 16.3$ & $41.8 \pm 17.2$ & $42.7 \pm 18.4$ \\
\hline
\end{tabular}

*Significant difference between stroke group and control group at 0.05 level

${ }^{a}$ Significant difference between spinner knob and steering handle at 0.05 level 


\section{DISCUSSION}

As the number of drivers with post-stroke hemiplegia grows, driving rehabilitation becomes more important. To help these drivers, occupational therapists need to analyze their driving performance. The aim of this study was to investigate and compare muscle activation differences between using a spinner knob and a steering wheel in drivers with post-stroke hemiplegia and non-disabled drivers. The experimental group was comprised of five drivers with poststroke, left-side hemiplegia. Because the required devices, driving position, and other factors may vary depending on the affected side, this study included only drivers with leftside hemiplegia.

When the drivers in the experimental group turned the vehicle to the right using a steering wheel or a spinner knob, the activation of the biceps brachii was significantly different. Mo et al. ${ }^{8)}$ reported that the biceps brachii was activated when a vehicle was turned to the right, and the anterior deltoid and the triceps were also activated when engaging in that movement. However, in this study, activation of the triceps and the anterior deltoid was not observed. With regard to the control motion of a spinner knob or a steering wheel when turning a vehicle to the right, the biceps brachii is considered to be the main action muscle. Therefore, only activation of the biceps brachii was found to be significantly different between using a steering wheel and a spinner knob. It is interesting to note that activation of the biceps brachii was not significantly different between using a steering wheel and a spinner knob in the control group, unlike drivers in the experimental group. This shows that a spinner knob requires less muscle activation than a steering wheel for drivers with stroke.

The results of the comparison of the activation of the biceps brachii between the two groups show that, the biceps activation in the drivers in the experimental group was lower than that in the control group. This could be because four of the five drivers in the experimental group usually used a spinner knob, while none of the drivers in the control group used a spinner knob. Adaptation might influence activation of the related muscles ${ }^{9}$.
Because drivers with stroke have difficulty with bilateral hand use, a number of them use a spinner knob instead of a steering wheel. Occupational therapists must know how to analyze a stroke patient's driving performance and understand how an adaptive driving device can help them to drive safely. The results of this study indicate that a spinner knob requires less activation of the main muscles than a steering wheel, especially in drivers who have had a stroke. The effect of adaptation could not be ruled out. Therefore, in the future, research on the time it takes to adapt to using a spinner knob and other adaptive devices will be needed. Limitations of this study are that all the participants in the experimental group were drivers with left hemiplegia, and the number of participants was insufficient to generalize the results. Also, all participants drove at different speeds. Although there was a speed limit for the driving, the drivers couldn't keep a consistent speed.

\section{REFERENCES}

1) Lee SY, Shim JM, Park MC: A study on the activation of forearm muscles during gripping by handle thickness. J Phys Ther Sci, 2011, 23: 549-551 [CrossRef].

2) Husain A, Khan AA, Hasan F: Ergonomic evaluation of effects of handle shape and task orientation on human performance in screw driving task. Int J Advance Tech, 2013, 4: 105-114.

3) Yoo WG: Effects of the different screwdriver handle sizes on the forearm muscles activities and wrist motion during screw-driving work. J Phys Ther Sci, 2013, 25: 885-886 [CrossRef]. [Medline]

4) Coleman Bryer R, Rapport LJ, Hanks RA: Determining fitness to drive: Neuropsychological and psychological considerations. In: Driver rehabilitation: Principles and practice. St Louis: Mosby, 2005, pp 165-185.

5) Kim KH, Son K, Yoo WS: Relationship between comfort and safety of sitting posture of a driver during vehicle crash. Trans Korean Soc Automat Eng, 2003, 11: 102-109.

6) Kim SS, Lee HS: Effects on the lumbar and quadriceps muscle activities when getting in and out of RV and passenger cars. J Ergon Soc Korea, 2009, 11: 195-199.

7) Park SJ, Kim CB, Kim CJ, et al.: Comfortable driving postures for Koreans. Int J Ind Ergon, 2000, 26: 489-497 [CrossRef].

8) Mo SM, Hyun YJ, Kim CS, et al.: Correlation between muscle contraction and vehicle dynamics in a real driving. In: Advances in affective and pleasurable design. Florida: CRC Press, 2013, pp 196-202.

9) Kloter E, Dietz V: Obstacle avoidance locomotor tasks: adaptation, memory and skill transfer. Eur J Neurosci, 2012, 35: 1613-1621 [CrossRef]. [Medline] 